FZMw Jg. 7 (2004) S. 69-92

\title{
Reinhard Keisers Orpheus-Opern - Anmerkungen zu den Libretti
}

\author{
von Arno Lücker
}

\section{Einleitung und Anmerkungen zur Chronologie}

$$
-1-
$$

Der Orpheus-Mythos gilt als das beliebteste Opernsujet überhaupt. Hinter dem Bekanntheitsgrad der zum (wissenschaftlichen wie künstlerischen) Standartrepertoire gehörenden Vertonungen Monteverdis und Glucks treten zahlreiche andere Werke aus verschiedenen Gründen zurück. Im Falle der norddeutschen Barockoper liegt der Grund vor allem in der schlechten Überlieferungssituation. Die aus dieser Zeit erhaltenen Textbücher allerdings bürgen für eine recht hohe regionale Popularität der OrpheusThematik, was nicht erst durch die Oper Telemanns ${ }^{1}$ und mehrere Libretti, die Reinhard Keiser als Vorlage dienten, bezeugt wird. Schon 1659 griff ein tragisches Geticht den Orpheus-Mythos "[...] zu sonderbahrem Gefallen Der [...] Frauen Sophia Elisabeth Vermähleter Hertzogin zu Braunschweig und Lüneburg" anlässlich ihres Geburtstages auf. $^{2} 1684$ soll dann in Salzdahlum Der beständige Orpheus ${ }^{3}$ - ebenfalls zu einem höfischen Fest - gespielt worden sein. ${ }^{4}$

$$
-2-
$$

Es wäre reizvoll, die mannigfaltigen 'Orpheus-Variationen' in der Musikgeschichte Norddeutschlands des 17. und frühen 18. Jahrhunderts einmal gesondert zu untersuchen. Hier allerdings sollen jene Libretti im Vordergrund stehen, die Reinhard Keiser als Vorlage für seine Orpheus-Opern verwendete.

\footnotetext{
$1 \quad$ Die wunderbare Beständigkeit der Liebe, oder ORPHEUS; [...] (Hamburg 1726). Vgl. Marx, Hans Joachim: Die Hamburger Gänsemarkt-Oper: Katalog der Textbücher (1678-1748). Hamburg: Laaber, 1995. Nr. 303.

2 ORPHEUS AUS THRACIEN DER CALLIOPE UND DESS APOLLINIS SOHN. [...] In Musicalische Noten übersetzet Von Johan Jacob Löwen. (Wolfenbüttel 1659). Vgl. Thiel, Eberhard: Libretti. Verzeichnis der bis 1800 erschienen Textbücher. (Kataloge der Herzog-AugustBibliothek Wolfenbüttel). Frankfurt/Main, 1970. Nr. 1207. [Die Textvorlage lieferte damals Anton Ulrich Herzog von Braunschweig.]

Thiel: Libretti. Nr. 351.

Als Komponist wird diesbezüglich Johann Rosenmüller vermutet, was jedoch einigermaßen unwahrscheinlich dünkt. Im übrigen ist das entsprechende Libretto äußerst selten Gegenstand musikwissenschaftlicher Untersuchungen geworden, lediglich McCredie führt es des öfteren in seiner vierzig Jahre alten Dissertation als Beispiel für häufig vorkommende musikalische Szenenanweisungen an. Siehe: McCredie, Andrew D.: Instrumentarium and instrumentation in the north german baroque opera, Hamburg: Diss., 1964.
} 
Bezüglich ihrer Chronologie sowie ihrer Überlieferung besteht innerhalb der musikwissenschaftlichen Literatur kein klares Bild. Ein Grund dafür dürfte sein, dass einige Autoren die Titel in stark verkürzter Form wiedergeben. Beispielsweise ist oft nicht klar, ob mit der Erwähnung des 'Orpheus von Reinhard Keiser' die einzig erhaltene musikalische Quelle $^{5}$ - welche tatsächlich mit Orpheus überschrieben ist oder eines der Libretti gemeint ist, die aber jeweils höchst unterschiedliche Titel tragen. Nur die Urfassung von 1698 heißt ebenfalls schlicht Orpheus. Ungenaue Angaben führen zu Irritationen, die aufgrund der wenig übersichtlichen Quellensituation doppelt problematisch sind. ${ }^{6}$

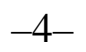

1698 wurde Keisers Orpheus in Braunschweig erstmals aufgeführt. ${ }^{7}$ Offenbar empfand man das Stück als zu lang, was bei der (für damalige Verhältnisse hohen) Blattanzahl des Librettos (45 Seiten) durchaus nachvollziehbar scheint. Aus der daraufhin geteilten Urfassung entstand eine Doppeloper. ${ }^{8}$ Der erste Teil hieß in der Braunschweiger Druckversion Die Sterbende EURIDICE ${ }^{9}$ (Braunschweig 1699) und in der Hamburger Fassung Die Sterbende EURYDICE Oder: ORPHEUS Erster Theil ${ }^{10}$ (Hamburg 1702). Dem zweiten Teil der Doppeloper gab man in Braunschweig den Titel DIE VERWANDELTE LEYER DES ORPHEUS ${ }^{11}$ (Braunschweig 1699), wohingegen der zweite Teil in Hamburg als ORPHEUS Ander Theil ${ }^{12}$ (Hamburg 1702) auftaucht. In Matthesons Musicalischem Patriot findet sich ein Nachweis der beiden Teile an 101. und 102. Stelle. ${ }^{13}$ Von ihm stammt auch die Information bezüglich der (schließlich wieder zusammengefügten) Fassung von 1709: "Orpheus, in eines gebracht, von Herrn Keiser und Bressand. Sonst wie oben [...], da es zween Theile waren."14 Schulze schreibt: "Mit dem 'sonst' ist offenbar vor allem gemeint, dass die Musik im ganzen

\footnotetext{
$5 \quad$ Mus.ms. 1486. Keiser: Orpheus. Staatsbibliothek zu Berlin - Preußischer Kulturbesitz. Musikabteilung mit Mendelssohn-Archiv. [Es handelt sich dabei um eine fast komplette Abschrift der Arien des Kopisten B.] Zur Quellensituation siehe: Haberkamp, Gertraut: Werke mit Musik für die deutschsprachige Bühne des 17. Jahrhunderts. Der aktuelle Quellenstand. In Teutschland noch gantz ohnbekandt: Monteverdi-Rezeption und frühes Musiktheater im deutschsprachigen Raum. Hg. Markus Engelhardt. Frankfurt/Main: Lang, 1994. S. 1-28. S. 1.

$7 \quad$ An der dortigen Hofoper gab es seit 1687 regelmäßige Vorstellungen.

8 Die Teilung einer als zu lang empfundenen Oper war durchaus üblich. Vgl. Haufe, Eberhard: Die Behandlung der antiken Mythologie in den Textbüchern der Hamburger Oper 1678-1738. Frankfurt/Main: Lang, 1994. S. 128.

9 Thiel: Libretti. Nr. 1527.

10 Marx: Katalog. Nr. 251.

11 Thiel: Libretti. Nr. 1685.

12 Marx: Katalog. Nr. 212.

13 Mattheson, Johann: Der musicalische Patriot [...]. Fotomechanischer Neudruck d. Ausgabe Hamburg 1728. Kassel: Bärenreiter, 1975. S. 185. 
dieselbe blieb." ${ }^{15}$ Die Fassung von 1709 kann aber schlecht von Keiser u n d Bressand stammen, schließlich starb Bressand bereits 1699. Das Problem des nicht genau bekannten Autors (bzw. Bearbeiters) der Fassung von 1709 wird noch zu diskutieren sein.

$$
-5-
$$

1709 entstand also eine aus beiden Teilen wiederum zusammengefügte Fassung mit dem Titel Die Biß in / und nach dem Todt / unerhörte Treue / Des ORPHEUS ${ }^{16}$ (Hamburg 1709), der man bisweilen auch als Treue des Orpheus in der musikwissenschaftlichen Literatur begegnet. Auf die Version des Jahres 1709 geht auch die einzig erhaltene musikalische Quelle zurück. ${ }^{17}$

$$
-6-
$$

Durch seine mehrmalige Beschäftigung mit dem Orpheus-Stoff wird Keisers Name häufiger in diesem Zusammenhang genannt. Dies führt bisweilen zu Verwirrung. Beispielsweise traf man bis vor kurzer Zeit im Katalog der Herzogin Anna Amalia Bibliothek Weimar auf ein Orpheus-Libretto von 1690. Als Aufführungsort wurde Braunschweig, als Komponist Reinhard Keiser angegeben. Die zweite Zuschreibung ist freilich nicht korrekt, Keiser war 1690 erst 16 Jahre alt und hielt sich wahrscheinlich als Thomasschüler in Leipzig auf. ${ }^{18}$ Von wem die Zuschreibung stammt, konnte nicht exakt geklärt werden. ${ }^{19}$

$$
-7-
$$

Es handelt sich bei betreffendem Orpheus-Libretto um die (gereimte) Übersetzung des L'Orfeo von Antonio Sartorio (Libretto und dt. Übersetzung: Aurelio Aurelij). Diese italienische Oper, die bereits 1673 in Venedig ihre Uraufführung hatte, wurde tatsächlich 1690 an der Braunschweiger Hofoper gespielt. ${ }^{20}$ Da Bressand 1690 schon ein Jahr in Braunschweig tätig war ${ }^{21}$, kann man annehmen, dass ihm das Libretto der Oper Sartorios vorlag. Wolff bemerkt: "Bressand hat in diesem Werk die bisherigen

15 Schulze, Walter: Die Quellen der Hamburger Oper (1678-1738). Eine bibliographisch-statistische Studie zur Geschichte der ersten stehenden deutschen Oper. Hamburg: Diss., 1938. S. 36.

16 Marx: Katalog. Nr. 51.

17 Quellenbeschreibung in: Schulze: Quellen. S. 106.

18 Roberts, John H: Art. Keiser, Reinhard. NGroveD. 2. Aufl. Hg. Stanley Sadie. Bd. 13. London: Macmillan, 2001. S. 448-455. S. 448. Zumindest aber ist die falsche Zuschreibung auf Hinweis des Autors aus dem Katalog entfernt worden.

20 Mischiati, Oscar: Art. Sartorio, Antonio. MGG. Bd. 11. Hg. Friedrich Blume. Kassel: Bärenreiter, 1963. Sp. 1418-20. Sp. 1418.

21 Marx, Hans Joachim: Art. Bressand, Friedrich Christian. MGG. Zweite, neubearbeitete Ausgabe. Hg. Ludwig Finscher. Autorenteil. Bd. 1. Kassel: Bärenreiter; Stuttgart: Metzler, 1999. Sp. 85961. Sp. 859 . 
italienischen Dramatisierungen zusammengefasst und durch reizvolle Einlagen (z. B. ein Ballett der Bienen) bereichert. ${ }^{22}$

$$
-8-
$$

Vergleicht man das Libretto der Oper Sartorios mit dem Bressandschen Textbuch, so wird wirklich deutlich, dass die Einfügung der Figur der Autonoe ${ }^{23}$ durch den Braunschweiger Hofdichter auf die Rezeption Aurelijs zurückgeht. Autonoe, die bei Bressand fast schon den Rang einer Hauptrolle einnimmt, ist laut Wolff tatsächlich eine reine Erfindung des italienischen Textdichters:

\begin{abstract}
"Francesco Buti gab der Sage [...] die Form eines Intrigenspiels zwischen Venus und Juno; er führte die Figur des Hirten Aristeo als Nebenbuhler des Orpheus [von Vergil] ein; sein Text wurde mit der Musik von Luigi Rossi vor allem in einer französischen Fassung bekannt: Le mariage d'Orphée et d'Euridice (Paris 1647) [...]. Der Venezianer Aurelio Aurelij behielt in L'Orfeo (Venedig 1673) nicht nur Aristeo bei, sondern gab diesem auch noch eine frei erfundene Geliebte Autonoe; der Stoff wurde hier zu einer barocken 'Tragikomödie der Eifersucht' [...]."24
\end{abstract}

\title{
$-9$
}

Dies trifft auch auf Bressands Version zu, dessen Handlung von Intrigen und Eifersüchteleien bestimmt wird. Es wäre schon allein erkenntnisreich, die Verarbeitung des Orpheus-Sujets bei Bressand genauer zu untersuchen, gerade weil in der oben erwähnten Oper von 1684 (Der beständige Orpheus) mit der Figur der Crinise ${ }^{25}$ eine im Vergleich zu Autonoe sehr ähnliche Intrigantin auftritt und bezüglich dieses Librettos daher ein Einfluss auf Bressand ebenfalls nicht auszuschließen ist.

$$
-10-
$$

Im Vordergrund dieser Arbeit steht jedoch ein - von der Musikwissenschaft bisher nicht vorgenommener - Vergleich der Orpheus-Libretti von 1698 (Braunschweig) und 1709 (Hamburg). Wie zu zeigen sein wird, sind die Gründe für die spezifischen Modifikationen, denen das frühere Libretto unterworfen war, in den sich wandelnden opernästhetischen Voraussetzungen $\mathrm{zu}$ suchen. Als Ausgangspunkt für die Gegenüberstellung wurden freilich besonders auffällige Unterschiede gewählt, anhand

\footnotetext{
Wolff, Hellmuth Christian: Orpheus als Opernthema. Musica XV (1961). S. 423-425. S. 424.

Autonoe ist die vernachlässigte Verlobte Aristeus'.

Wolff: Orpheus als Opernthema. S. 423.

Crinise ist ebenfalls heimlich in Orpheus verliebt.
} 
derer die Änderungen der ursprünglichen Braunschweiger Fassung analysiert werden sollen.

\section{Gesprochene Dialoge in der Orpheus-Urfassung?}

$-11-$

Um 1700 waren eine ganze Reihe von Gattungsbezeichnungen für Bühnenwerke im Umlauf, die "[...] abwechselnd und ohne jeden Unterschied gebraucht [wurden]."26 Vorrangig begegnet man der Bezeichnung Singe=Spiel, so beispielsweise im Titel der von uns betrachteten Textbücher (1698 und 1709). Schon aber ab etwa 1694 beginnt sich die Gattungsbezeichnung Opera immer mehr durchzusetzen. ${ }^{27}$ Als andere Bezeichnungen sind beispielsweise Musicalisches Schau=Spiel ${ }^{28}$, Musicalisches Trauer $=$ Spiel $^{29}$ oder Musicalisches Lust $=$ und Tant $=$ Spiel $^{30} \mathrm{zu}$ nennen.

$$
-12-
$$

Die Überschrift Singe=Spiel besagt jedoch nicht unbedingt, dass Dialoge, wie im 'Singspiel' des späten 18. und des 19. Jahrhunderts, ge s prochen wurden. Die verschiedenen Gattungsbenennungen lassen allgemein $\mathrm{zu}$ dieser Zeit keinen Rückschluss auf die Dialogfrage zu. ${ }^{31}$ Wolff schreibt allerdings: "Unmöglich erscheint es keineswegs, dass die Dialoge teilweise gesprochen wurden, denn die Hamburger Oper übernahm zahlreiche Elemente aus den Schauspielaufführungen jeder Art und verband überhaupt die heterogensten Dinge miteinander." ${ }^{32}$

$$
-13-
$$

Als einen Beweis für die Existenz gesprochener Dialoge innerhalb von Opern sieht Wolff die entsprechenden Kommentare des Librettos an. So heißt es beispielsweise in Michal und David ${ }^{33}$ (1679) zweimal ausdrücklich, dass g e s p r o c h e n werden soll. Solche Szenen seien aber die Ausnahme gewesen. Die Reimform lasse dabei nicht zwingend auf einen gesungenen Vers schließen, gereimte Dialoge fände man schließlich auch in außermusikalischen Bühnenwerken der Zeit. Zudem bemerkt Wolff, dass es

\footnotetext{
26 Wolff, Hellmuth Christian: Die Barockoper in Hamburg (1678-1738). Wolfenbüttel: Möseler, 1957. S. 226.

Ebd.

28 z. B. L'AMORE VERSO LA PATRIA [...] von Feind und Keiser (aufgeführt 1711). Vgl. Marx: Katalog. Nr. 18. z. B. Der Fall Des grossen Richters in Israel / Simson [...] von Feind und Graupner (aufgeführt 1709). Vgl. Marx: Katalog. Nr. 103. z. B. Das Römische April=Fest. von Feind und Keiser (aufgeführt 1716). Vgl. Marx: Katalog. Nr. 232. Wolff: Barockoper. S. 226.

Ebd. S. 227.

Die Wol und beständig=liebende MICHAL Oder Der Siegende und fliehende David. [...] von Elmenhorst und Franck (aufgeführt 1679). Vgl. Marx: Katalog. Nr. 300.
} 
sogar als legitim angesehen wurde, Operntexte vollständig gesprochen aufzuführen, worin er bis heute zumindest nicht widerlegt worden ist. Resümierend meint Wolff: "Es besteht also die Möglichkeit, dass ein Teil der Dialoge in den Aufführungen der frühen Hamburger Oper gesprochen worden ist." ${ }^{34}$

Vergleicht man daraufhin die hier im Mittelpunkt stehenden Orpheus-Libretti von 1698 und 1709, so stellt man fest, dass einige Passagen im früheren Libretto mit Anführungszeichen am Anfang jeder Zeile versehen sind. Möglicherweise dienten sie zur Kennzeichnung gesprochener Sätze. Da Wolff als Beleg für seine oben zitierte These nur Bühnenwerke des Zeitraums 1679-1695 anführt, darf man davon ausgehen, dass diese Praxis 1709 nicht mehr bestand.

$$
-15-
$$

Und tatsächlich sind gerade die in Anführungszeichen gesetzten Teile der früheren Version vollständig gestrichen worden. ${ }^{35}$ Es handelt sich dabei beispielsweise um die pastorale Szene, in der sich Eurydice mit einigen Nymphen tanzend die Zeit vertreibt (III, 8). Bei einer Art Fangspiel rufen sich die Parteien gegenseitig Verse zu. Dass hier tatsächlich nicht gesungen, sondern gesprochen (bzw. gerufen, geflüstert etc.) wurde, ist wahrscheinlich. Auch Wolff nimmt an, dass dies insbesondere bei "[...] realistische[n] Volksszene $[n][\ldots]^{\prime 36}$ der Fall gewesen sein könnte. Ein weiterer bestätigender Hinweis ist die Szenenanweisung des folgenden Auftritts (III, 9), hier heißt es: "Aristceus gantz ängstig sich anstellend / $r$ uft von fernen" (Hervorhebung von mir). Aufgrund der Konsequenz der Bearbeitung kann man annehmen, dass die frühere Fassung tatsächlich noch gesprochene Dialoge enthielt. Die (freilich latente, weil nur durch Nennung einzelner Werke kenntlich gemachte) zeitliche Eingrenzung der opernspezifischen Sprech-Praxis bei Wolff (1679-1695) ließe sich angesichts des Braunschweigischen Librettos ergo auf das Jahr 1698 ausdehnen.

Wolff: Barockoper. S. 228.

Eine vollständige Auflistung der Änderungen findet sich in Tab. I.

Wolff: Barockoper. S. 228. 
Tab. I: Streichungen von gesprochenen Dialogen (1698)

\begin{tabular}{c|l} 
Ort der Streichung & betreffende Person(en) \\
\hline I, 2 & Orpheus, Cleon \\
\hline I, 4 & Thya, Eine Bacchantin, Chor, Orpheus \\
\hline I, 6 & Thya \\
\hline I, 7 & Thya, Autonoe \\
\hline II, 1 & Dimas \\
\hline II, 2 & Eurydice, Aristeus \\
\hline II, 6 & Orpheus, Autonoe \\
\hline II, 9 & Autonoe \\
\hline III, 3 & Thya \\
\hline III, 7 & Orpheus, Eurydice \\
\hline III, 8 & Eurydice, Die Nymfen, Nomia, Chor der Nymfen \\
\hline III, 9 & Aristeus, Autonoe \\
\hline IV, 9 & Ein Elyseischer Geist, Ein anderer Elyseischer Geist, Chor der Elyseischen Geister
\end{tabular}

$-16-$

Offenbar sah Keiser also 1709 die ursprüngliche Vorlage Bressands - allein schon aufgrund der in ihr noch enthaltenen Dialogszenen - als veraltet an. Bestätigt wird dies durch die Aussage Wolffs, der meint, gerade Keiser hätte großen Wert auf die Rezitative - nicht also auf gesprochene Dialoge - gelegt. ${ }^{37}$ Das wird von dem Librettisten Barthold Feind emphatisch zugesichert:

\begin{abstract}
"Ein Semicolon, Punctum, Signum interrogandi, Exclamationis, Colon und Comma hat seine Gesetze und Cadence, die / wie Feuer und Wasser / differiren / und wenn ein Acteur einen Brief singend abließt in einer / von einem exquisiten Musico verfertigten / Opera, wie von Reinhard Keysern / so wird man fast ein tertium quid unter Singen und Sprechen bemercken / welches man vom gantzen Recitatif sagen muss; [...]."
\end{abstract}

$$
-17-
$$

Die Streichung der gesprochenen Dialoge hing nicht nur mit der Langatmigkeit Bressandscher Libretti zusammen, die dem Autor, Übersetzer und Musikdirektor

\footnotetext{
$37 \quad$ Ebd. S. 226.

38 Feind, Barthold: Deutsche Gedichte. Faksimiledruck der Ausgabe von 1708. Hg. W. Gordon Marigold. Bern: Lang, 1989. S. 78.
} 
Braunschweigs des öfteren vorgeworfen wird. ${ }^{39}$ Die Kürzung des Textbuchs kann vielmehr als eine Aktualisierung für die aufstrebende Gänsemarktoper in Hamburg interpretiert werden. Hinter den Ansprüchen eines Publikums, das in Hamburg zunehmend nach kurzweiliger Darbietung (möglichst italienischer) Affektarien verlangte, trat die Handlung (die Bressand in der Fassung von 1698 noch im Stile des französischen Dramas weit auffächerte ${ }^{40}$ ) zurück.

$$
-18-
$$

Es handelt sich bei den aus dem Libretto von 1698 gestrichenen Teilen daher auch primär um solche, in denen nur be richtet wird. Besonders deutlich wird dies, wenn man die Auftritte III-VI der vierten Handlung betrachtet. Diese Auftritte wurden deshalb komplett gestrichen, weil in ihnen fast ausschließlich von Taten und Begebenheiten die Rede ist:

$$
-19-
$$

IV, 3: Facus e r z äh $1 \mathrm{t}^{41}$ Pluto, wie er Eurydice unter den Toten traf und sie ihm als die Einzige voll "Zucht und Tugend" erschien.

IV, 4: Rhadamantus be ri ch te t Pluto: "Ach! Pluto / das geschick erzeigt sich unsern feind / ein Sterblicher / der noch im leben / der noch den geist nicht aufgegeben / ist mit erschrecklicher Vermessenheit in unser Reich gebrochen / [...]."

IV, 5: Ascalaphus e rzählt, wie Orpheus sich bei seinem Eindringen in die Hölle weder durch "[...] furcht noch grimm erwecken [...]" ließ, lediglich bei seinem Gang durch das Reich der Toten auf seinen Saiten spielte.

IV, 6: Orpheus - inzwischen vor dem Thron des Höllengottes angelangt b e r i c h t e t Pluto sein Leid, das ihn den Gang in die Hölle wagen ließ.

$$
-20-
$$

Bressand zeigt sich hier den französischen Dramatikern sehr nahe. Berichte und erzählte Handlungen waren aber um 1709 in Hamburg offenbar nicht mehr gefragt, was an der Distanzierung Feinds vom Vorbild der Franzosen deutlich wird:

"Wo sonst keine Affecten sind / da sind auch keine Actiones, und wo keine Actiones sind / da wird es auf dem Theatro sehr frieren. Je natürlicher der Poet sich eine Idée von der vorzustellenden Sache und Affect macht / und je

\footnotetext{
39 Degen meint beispielsweise, die Hauptschwäche Bressands sei die Langatmigkeit seiner Textbücher gewesen. Vgl. Degen, Heinz: Friedrich Christian Bressand. Braunschweig: Diss. Teildruck, 1935. S. 49.

40 Dies resultierte aus der Beschäftigung mit Trauerspielen eines Molières, Racines oder Corneilles, die Bressand teilweise übersetzte. Vgl. Marx, Hans Joachim : Art. Bressand, Friedrich Christian. MGG. Zweite, neubearbeitete Ausgabe. Hg. Ludwig Finscher. Autorenteil. Bd. 1. Kassel: Bärenreiter; Stuttgart: Metzler, 1999. Sp. 859-61. Sp. 861.

41 Hervorhebungen jeweils von mir.
} 
genauer er denselben in dem nohtwendig dazu erforderten mouvement d'esprit bey sich überleget / die Umstände reiflich erweget / und wo keine sind / welche erdichtet / je besser wird der Affect seyn. Aus dieser Ursach bin ich mit den Frantzsösichen Tragicis, die darinnen die alten Griechen und Lateiner imitiren / nicht einerley Meynung / dass sie in den Tragedies die fürnehmsten Thaten nur erzehlen lassen / so / dass die fürnehmste Action des Acteurs in einer wehmütigen Erzehlung allein besteht / [... ." 42

$$
-21-
$$

Demnach wären also die oben aufgezählten Auftritte bei Feind auf Ablehnung gestoßen. Inwieweit sich seine Äußerung mit der Meinung des Hamburgischen Publikums deckt, ist zwar nicht genau festzustellen, da Feind aber als Librettist höchst erfolgreiche Opernvorlagen lieferte, kann davon ausgegangen werden, dass dem Publikum um 1709 bei der Aufführung der Fassung von 1698 tatsächlich unwohl geworden wäre. Bestärkt wird diese These über das Verhältnis des Autors zum Publikum durch die Ansicht Eberhard Haufes, der in seiner Arbeit erstmals der Analyse der Vorberichte nähere Aufmerksamkeit schenkte. Er bemerkt: "[...] Letztlich waren die oft so umfangreichen Vorworte der Textbücher im ganzen eine Spiegelung dieses Verhältnisses [...]"43 und die Tatsache, dass Feind selbst bald gar keine mythologischen Opernvorlagen mehr schrieb, zeige nur, so Haufe, "[...] wie bald sich das Interesse an dieser Gattung zu verringern begann." 44 Wenn mythologische Opern von Erfolg gekrönt sein wollten, so mussten sie also (1709 mehr denn je), so fasst es Zelm zusammen, "Affekte erzeugen, Aktionen vorstellen [und] Maschinen und Veränderungen veranlassen." 45

\section{Die "vermischte Schreib-Art dazumahlen in Hamburg"}

$-22-$

Die frühe Hamburger Oper zeigte von Anfang an Ähnlichkeiten mit der Opera in Venedig. Sie glich dem italienischen Vorgänger nicht nur in dem genossenschaftlichen Pachtsystem und der öffentlichen Zugänglichkeit, früh orientierte man sich auch an bestimmten musikalischen Stilcharakteristika und den Sujets der Opern. Beispielsweise übersetzte man häufig die italienischen Textbücher als Vorlage für eigene

Feind: Gedichte. S. 106.

Haufe : Mythologie. S. 262.

Ebd. S. 104.

Zelm, Klaus: Die Opern Reinhard Keisers. Studien zur Chronologie, Überlieferung und Stilentwicklung. München: Katzbichler, 1975. S. 27. 
Bühnenwerke. Das Modediktat Frankreichs und Italiens war für Feind Anlass zur Kritik. Im Vorbericht zu der Oper La costanza sforzata ${ }^{46}$ schreibt er:

\begin{abstract}
"[...] Denn so lange solche [diejenigen, die Schauspiele verschiedener Nationen gesehen und gelesen haben] ihre Curiosité mit nichts neues befriedigen können / und nur immer Sachen finden / die bey den Italiänern und Frantzosen schon abgedroschen / wird den Teutschen kein grosser Ruhm zuwachsen."
\end{abstract}

$$
-23-
$$

Der Librettist und Jurist forderte in den Vorberichten seiner Textbücher und vor allem in den Gedancken von der Opera nachdrücklich die Emanzipation der deutschen Opernkunst und trat Modezwängen (wie der Einfügung lustiger Personen in ernste Stücke), sowie der Praxis der Übersetzung fremdsprachiger Operntexte entschieden entgegen. ${ }^{48}$ Der italienischen Sprache gestand er freilich trotzdem Vorteile zu:

"Den Italiänern favorisiret ihre Sprache hauptsächlich darinnen / dass sie ein Ding kürtzer geben können / als wir / welches dem Musico im Recitativ ungemeinen Vortheil zu wege bringet [...] Sie dürfen dabey sich nicht / wie wir / an eine gezwungene Construction binden / und in ihrem Metro ihre Absicht samt den Frantzosen / Portugisen / Spaniern / Lateinern und Griechen auf den Accent der Wörter und Reime richten / welches ihnen kein geringer Vortheil." 49

$$
-24-
$$

Man könnte meinen, dass gerade jener Librettist, der in diesem Vorwort noch die Loslösung von italienischen und französischen Leitbildern forderte, selbst mit gutem Beispiel vorangehen wollte. In La costanza sforzata finden wir jedoch noch zehn italienische Arien. Trotz aller Kritik an der nahen Orientierung an ausländischen Vorbildern rechtfertigt sich Feind in keinem Vorbericht seiner Libretti für die Verwendung dieser Texte, was daher schlicht für die zunehmende Akzeptanz dieser Praxis spricht.

\footnotetext{
$46 \quad$ La COSTANZA SFORZATA Die Gezwungene Beständigkeit Oder Die listige Rache Des SUENO. Musik von Keiser (aufgeführt 1706). Vgl. Marx Katalog. Nr. 68.

$47 \quad$ Vorbericht zur o. a. Oper. Feind: Gedichte. S. 334.

48 Wolff: Barockoper. S. 44.

49 Feind: Gedichte. S. 334-35.
} 


\section{Zur Einfügung italienischer Arien in das Hamburger Libretto}

$$
-25-
$$

Vergleicht man daraufhin die Libretti von 1698 und 1709 miteinander, so fällt die Einfügung italienischer Arien in die spätere Version auf. Es soll nun gezeigt werden, wie der Autor dieser Arientexte bei der Einfügung verfuhr, wobei auch jeweils die Handlung des Librettos mit aufgegriffen wurde, damit die Gründe für die Hereinnahme des fremdsprachigen Arientextes deutlich werden.

$$
-26-
$$

Die ersten zwei italienischen Arien finden sich im letzten Auftritt der ersten Handlung. ${ }^{50}$ Hier wurden nicht - wie man vermuten könnte - deutschsprachige Arien gestrichen, sondern lediglich italienische Textteile hinzugefügt. So erscheint der letzte Auftritt dieser Handlung mit Affekt geradezu überfüllt, er beginnt zunächst mit einer deutschen Duo-Arie (Angenehmste Liebes Kertzen) in der Orpheus und Eurydice ihre ewige Liebe bekunden und endet nach kurzem Rezitativ mit zwei Solo-Arien in italienischer Sprache: Zunächst singt Eurydice die Arie Dolce mia vita, dann entgegnet ihr Orpheus Alma del core Spirto de l'alma. Die inhaltliche Aussage ist in allen drei Arien ähnlich. Es scheint, als genüge die bloße A n w e n d u ng der italienischen Sprache, um den Affektgehalt der Arien zu erhöhen. Diese Vermutung wird dadurch bestätigt, dass die beiden italienischen Arien das Finale der ersten Handlung bilden.

$$
-27-
$$

Im zweiten Auftritt der zweiten Handlung treffen wir auf die dritte Arie in italienischer Sprache (Vittoria mio core). Eurydice singt erneut von beständiger Liebe (Costanza in amore), Aristeus - heimlich in die Verlobte seines Halbbruders verliebt - versucht Orpheus schlecht zu machen, was bei Eurydice jedoch auf taube Ohren stößt. Anders als in der ersten Handlung wurde hier allerdings die deutsche Arie des Librettos von 1698 gestrichen. Die Ähnlichkeit mit dem italienischen Text lässt jedoch auf eine Neubearbeitung schließen, die speziell für die Orpheus-Oper in Hamburg geschrieben worden sein könnte:

\footnotetext{
50 „Handlung“ ist der damals regional verbreitete opernspezifische Begriff für „Akt“.
} 
1698 (II, 2):

Sey zufrieden/mein liebendes herze/

Dein Vergnügen bricht nun heran/

Dich begleiten die lachenden Scherzel

da dir Hymnens erfreuliche Kerzel

zeigt zu voller Vergnügung die bahn.

Sey zufrieden \&c.
1709 (II, 2):

Vittoria mio core!

Costanza in amore

Trionfa, si si.

Se stringo il mio Bene,

Son dolci le pene,

Mi basta cosi.

Vittoria \&c.

$-28-$

Die vierte Einfügung wurde im dritten Auftritt (2. Handlung) vorgenommen (A vincer $i$ sensi): Aristeus hat Eurydice seine Liebe gestanden. Sie rät dem Verliebten jedoch, sich nicht allzu sehr seinen Gefühlen hinzugeben. Anstelle der Arie finden sich im Libretto von 1698 lediglich fünf normale Textzeilen. Die Arie wurde also zur Unterstreichung der Aussage Eurydices speziell eingefügt.

$$
-29-
$$

Die folgende Änderung, die im Libretto des Jahres 1709 auftaucht, gleicht dem zweiten Beispiel. Autonoe, die Verschmähte des Aristeus, macht sich hier immer noch Hoffnungen auf eine Rückkehr ihres Gatten. Auch hier ähneln sich die Aussagen der Arientexte:

1698 (II, 5):

Werthe Hoffnung/ bleibe doch/

scheide nicht aus meinem herzen;

Du erleichterst mir mein Joch/

du nur stillst mir meine schmerzen.

Werthe \&c.
1709 (II, 5):

Cara, e dolce speranza,

Non mi lasciar, nò, nò;

Premia la mia costanza,

E lieto poi sarò.

Cara \&c.

Es war nach 1703 in Hamburg üblich, die italienischen Arien mit einer deutschen ProsaÜbersetzung zu versehen, die parallel ins Textbuch gedruckt wurde. Merkwürdig erscheint es allerdings, daß im Libretto von 1709 der italienische Text dieser Arie nochmals in eine deutsche Prosa-Übersetzung gebracht wurde, wo doch ein gereimter Text aus dem Jahre 1698 bereits zur Verfügung stand. Vielleicht wurde dies aber bei der Neubearbeitung für die Aufführung 1709 schlichtweg nicht berücksichtigt, schließlich stellen ohnehin nicht alle Arien gereimte Übersetzungen ins Italienische dar. 
Es folgen nun acht Auftritte ohne eingefügte italienische Arie. Im vierten Auftritt der dritten Handlung (1698: III, 6) findet sich zu Beginn erneut eine Arie der Autonoe, die einen deutlichen Lamento-Charakter besitzt (Dove sei). Sie wurde nachträglich in das Libretto eingefügt und dient wiederum der Verstärkung des Affekts.

$$
-31-
$$

An der Stelle der nächsten Einfügung soll Orpheus sich in Aristeus' Bienengarten einfinden, weil dieser Eurydice während der Abwesenheit des Orpheus entführen will. Das Paar drückt seinen Abschiedsschmerz in einer italienischen Duo-Arie aus (Chi non spera in amor, III, 5).

$$
-32-
$$

Eurydice ist, als sie vor Aristeus flüchten wollte, von einer Schlange gebissen worden. Der Schuldige macht sich deswegen nun Vorwürfe und besingt seinen Schmerz in einer Aria (Ch mi toglio il mio tesoro?), die wir im siebten Auftritt der dritten Handlung finden. Auch im Libretto von 1698 (dort: III, 11) zeigt sich Aristeus verständlicherweise nicht erfreut ( $O$ unglückseel'ge Stund!), es findet sich aber keine deutsche Version dieser oder einer ähnlichen Arie. Nur in der Version von 1709 wird die dramatische Situation explizit zur Affektdarstellung der Trauer genutzt.

$$
-33-
$$

Auch die nächste in das Libretto von 1709 eingefügte italienische Arie wird von Aristeus gesungen (IV, 5). Dem Unglückseeligen wurde gerade die Nachricht von der Wanderung des Orpheus berichtet, der seiner Eurydice in die Hölle gefolgt ist. Er ist zwischen Hoffnungs- und Hassgefühlen hin- und hergerissen (Da tempesta di pensieri). In der gestrichenen Arie des Librettos von 1698 kommt allerdings eher die völlige Hoffnungslosigkeit und das Spiel mit Selbstmordgedanken zum Vorschein (Gib mir zurück mein Leben/ damit ichs auf kan geben/ [...]; aus: III, 16), als der Gefühlssturm der italienischen Arie.

$$
-34-
$$

Nun folgen zwölf Auftritte ohne die Einfügung einer italienischen Arie. Die Szene, in der Orpheus vor Charon Harfe spielt, findet sich beispielsweise nur in der Version von 1698. In diesem Teil wurden die umfangreichsten Kürzungen vorgenommen. Die Abschnitte, in denen Orpheus die Tiere, Flüsse und Steine dazu bewegt, ihm bei seinem Klagegesang zuzuhören sind auch im Libretto von 1709 enthalten. Hier wurde jedoch keine italienische Arie eingefügt, was vielleicht verwundern könnte, da diese Situation eine Affektdarstellung in italienischer Sprache ja geradezu heraufbeschwört. Offensichtlich war die musikalische Ausarbeitung dieser Szenen aber in der früheren 
Version auf großen Zuspruch gestoßen, so dass der Bearbeiter des Textbuchs eine Modernisierung durch die Hinzufügung italienischer Arien nicht für nötig hielt.

$$
-35-
$$

Die vorletzte italienische Arie finden wir im neunten Auftritt der fünften Handlung. Hier besingt Autonoe ihren Kummer um Aristeus, der noch immer nicht zu ihr zurückgekehrt ist. Sie wünscht wieder Frieden und Ruhe zu finden (Cara pace, dolce calma). Die Aussage der deutschen Arie von 1698 (Ach! mein glück/ wenn wirstu scheinen nach so langen Unglücks=plagen?) ähnelt der italienischen Aria aber eher weniger frappierend.

$$
-36-
$$

Die letzte Einfügung finden wir im vorletzten Auftritt der fünften Handlung. Hier bittet Aristeus den Gott Phœbus um Erbarmen, was zwar im sechsten Auftritt der letzten Handlung der Version von 1698 ebenfalls, aber nicht mittels einer Arie geschieht.

\section{Die Frage des Urhebers der italienischen Arien}

$$
-37-
$$

Weder die spezifische musikwissenschaftliche Literatur noch die Libretti (Vorberichte, Titel etc.) erteilen eindeutige Auskünfte zur Person des Verfassers der italienischen Arien, so dass man den Librettisten selbst als Autor annehmen könnte. Im Falle der ersten deutschen Oper, in der italienische Arien vorkamen (Claudius, 1703, Musik von Keiser) war dies Heinrich Hinsch. Friedrich Chrysander geht jedoch - bezugnehmend auf dieses Werk - davon aus, dass es Keiser war, der als Erster italienische Arien in deutsche Libretti einfügte:

"Für uns ist der Claudius denkwürdig als der erste Hamburgische
Operntext, in welchem italienische Arien gestreut wurden, und Reinhard
Keiser hat den traurigen Ruhm, solches ins Werk gesetzt zu haben. Die Art,
wie Hinsch [der Textdichter] davon spricht, zeigt deutlich, dass Keiser es
war, welcher dieses neue Reizmittel ersann; zum Lohne dafür wurde er auch
sofort unter Deutschen als italienischer Tonsetzer gefeiert." ${ }^{51}$ [Anm. d.
Verf.: Offenbar nimmt Chrysander hier Bezug auf die Tatsache, dass sich

$51 \quad$ Chrysander, Friedrich: Geschichte der Hamburger Oper unter der Direction von Reinhard Keiser (1703-1706). AMZ XV. Jahrgang, Nr. 2-6 (1880). S. 1-41. S. 17-18. 
Reinhard Keiser auch italienisiert Rinardo Cesare ${ }^{52}$ oder Rinaldo Cesare (33 $^{53}$ nannte bzw. genannt wurde.]

$$
-38-
$$

Chrysander spricht hier einen Abschnitt in Hinschs Schrift Theatralische, geistliche, vermischte und galante Gedichte (Hamburg und Leipzig 1713) an, in der sich der Librettist für die Hereinnahme italienischer Arien quasi 'entschuldigt':

\begin{abstract}
"Da dann in den Theatralischen Gedichten / wegen der Italiänischen Arien / welche in der Opera Diana beybehalten / alle Liebhaber der Teutschen Sprache versichere / dass es mit meinem grösten Verdruß geschehen; Weil aber eine solche vermischte Schreib-Art dazumahlen in Hamburg schon als eine langhergebrachte Gewohnheit eingerissen / wuste ich es im Anfange nicht zu hintertreiben / wie mich auch ins künftige nichts / als ein höherer Befehl wieder dazu bewegen." 54
\end{abstract}

$$
-39-
$$

Ist Mattheson zu glauben, so kann Keiser selbst wohl kaum die italienischen Arien geschrieben haben. Zwar lobt der Autor in der Ehrenpforte seinen Kollegen, was die "[...] vernünftige Weise einen Text unter die Noten zu legen [...]" angeht, doch schränkt seine Würdigung durch eine Bemerkung zu den Sprachkenntnissen Keisers gleich wieder ein:

"Er hatte aber, bey weniger Lesung guter dahin gehöriger Bücher, und wegen Abgangs die hiezu nöthigen fremden Sprachen, theils auch aus Bequemlichkeit, die Gabe nicht, seine sonst sehr gesunde Gedancken in eine ordentliche Kunstform oder systematisch zu Papier zu bringen. Das überließ er also meinem Fleisse; [...]." ${ }^{\text {55 }}$

\footnotetext{
52 Mattheson, Johann: Grundlage einer Ehrenpforte [...]. Vollständiger, originalgetreuer Nachdruck mit gelegentlichen bibliographischen Hinweisen und Matthesons Nachträgen. Hg. Max Schneider. Graz: Akad. Dr. - u. Verl.-Anst., 1969. S. 125.

53 Eitner, Robert: Art. Keiser, Reinhard. Biographisch-Bibliographisches Quellen-Lexikon der Musiker und Musikgelehrten christlicher Zeitrechnung bis Mitte des neunzehnten Jahrhunderts. Hg. Robert Eitner. Leipzig: Breitkopf \& Haertel, 1901. S. 334-338. S. 334. Zitiert in: Haufe: Mythologie. S. 101. Mattheson: Ehrenpforte. S. 129.
} 
$\mathrm{Ob}$ es sich hier tatsächlich um eine grundsätzliche Aussage $\mathrm{zu}$ den Fremdsprachenkenntnissen Keisers oder - wie offenbar nicht selten - um persönliche Eitelkeit Matthesons handelt, kann nicht eindeutig gesagt werden.

Weiterhin könnte man vermuten, dass Keiser oder Hinsch fremde Arientexte für die Orpheus-Version von 1709 benutzt haben. Leichtentritt, der im übrigen annimmt, dass Keiser die italienischen Arien tatsächlich selbst in den Claudius einfügte, schreibt zu dieser Form der Textanleihe:

"Ob die italienischen Texte immer vom Komponisten hinzugethan wurden, wie hier, und nicht vom Textdichter stammten, lässt sich kaum mehr feststellen. Dagegen scheint sicher, dass sie aus italienischen Opern ohne weiteres hinübergenommen wurden, und wohl nur selten, vielleicht nie für das betreffende Stück gedichtet wurden, - den Hamburger Textverfertigern kann man schwerlich eine solche Beherrschung des Italienischen zutrauen. Es findet sich schon bei oberflächlicher Untersuchung eine Reihe von italienischen Arientexten, die in ganz verschiedenen Opern genau oder sehr ähnlich wiederkehren. Würde man eine grosse Zahl Operntexte der Epoche daraufhin untersuchen, so würden sich wahrscheinlich eine Reihe von Lieblingsarientexten der Komponisten aufstellen lassen. Bei ihrem meist allgemeingültigen Inhalt von Jubel oder Trauer, Liebe oder Hass ließen sich diese Texte leicht überall einschieben." 56

$$
-41-
$$

Leichtentritt irrt, was die Italienischkenntnisse der Hamburger Librettisten angeht. Beispielsweise waren sowohl Hinsch als auch Feind durchaus dieser Sprache mächtig. ${ }^{57}$ Auch mit dem verallgemeinernden Argument, italienische Arien seien vielleicht nie speziell für deutsche Textbücher geschrieben worden, scheint der Autor der ersten Dissertation über Keisers Opern falsch zu liegen, denn wir sahen, dass die italienischen Arien im Hamburgischen Orpheus, den gestrichenen deutschen Arien in der Textaussage sehr ähneln. Eine direkte Übernahme bereits vorhandener Arientexte scheint daher unwahrscheinlich. Wolff wies 1961 darauf hin, dass Bressand sich an dem

$56 \quad$ Leichtentritt, Hugo: Reinhard Keiser in seinen Opern. Ein Beitrag zur Geschichte der frühen deutschen Oper, Berlin: Diss. Teildruck, 2. Teil mit einem Band musikalischer Anlagen ist nie erschienen, 1901. S. 26.

57 Hinsch beherrschte neben Italienisch noch die französische Sprache, wie Wolff (Barockoper. S. 209) aufzeigt. Feind soll gute Kenntnisse des Französischen, Italienischen und vielleicht sogar des Spanischen besessen haben. Vgl. Marigold, Gordon W: Einleitung zu Feind Gedichte. S. 19. 
L'Orfeo-Libretto des Venezianers Aurelio Aurelij (Musik: Antonio Sartorio) orientierte. ${ }^{58}$ Da diese Oper - wie bereits in der Einleitung angesprochen - 1690 in Braunschweig gespielt wurde, könnte man (ausgehend von dem Argument Leichtentritts) vermuten, dass Keiser sich aus dem italienischen Libretto bediente, weil ihm der Textdruck, den Bressand einst besessen haben muss, möglicherweise zur Verfügung stand. Dies bestätigt sich bei einem Vergleich der italienischen Arien aus dem Libretto von 1709 mit dem Braunschweiger Textbuch von 1690 jedoch nicht. Leichtentritt verallgemeinert also, was sicherlich mit seiner Negativwertung der Italienisierungspraxis zusammenhängt.

$$
-42-
$$

Dass Keiser (im Vergleich zu Telemann oder Händel) vorzeitig in Vergessenheit geriet, ist nämlich nicht ausschließlich der schlechten Quellenlage zuzuschreiben. Zelm weist in seiner Dissertation aus dem Jahre 1975 darauf hin, dass negative Urteile von verschiedenen Autoren wie Chrysander, die teilweise auf das ausschweifende Leben Keisers und zum Teil auch auf die Heroisierung Händels in Abgrenzung zu Keiser abzielten, sich auf die Bewertung seiner Werke niederschlugen. ${ }^{59}$ Ein anderer Grund für die Tatsache, dass erst im späten 20. Jahrhundert eine Keiser-Renaissance einsetzte, mag sein, dass er - latent oder auch ganz offen - für den Niedergang der Gänsemarktoper verantwortlich gemacht wurde.

$$
-43-
$$

Leichtentritt hält sich bei der Bewertung der oben beschriebenen Praxis noch weitgehend zurück, während andere Autoren, wie beispielsweise Chrysander, wesentlich härter mit Keiser ins Gericht gehen. Hans Joachim Moser - allgemein zur Übertreibung und zum Anachronismus neigend - schreibt in seiner Musikgeschichte in hundert Lebensbildern (sich selbst zitierend): "'Claudius' (1703) [huldigte] zuerst dem Unfug, in ein völlig deutsches Stück italienische Arientexte mit ihren glatten Sprüchen einzuschieben, auf die es sich so bequem tirilieren ließ."60

Moser übernahm hier offensichtlich unreflektiert die Meinung Chrysanders und spitzte sie in der Art der Formulierung noch zu, obwohl Keiser mit der Einfügung italienischer Arien lediglich dem Hamburgischen Geschmack so gut entsprach, dass seine Opern selbst in Zeiten, in denen er sich nicht in Hamburg aufhielt, gespielt wurden. Ohne die enge Orientierung an den Forderungen des Hamburgischen Publikums, denen Keiser zusammen mit seinen Librettisten nachkommen wollte, wäre die Periode der 
Gänsemarktoper (nach anfänglichen Konzessionen an den Hamburgischen Klerus) nie zu einer der "[...] glanzvollsten Epochen des deutschen Theaters [...]"61 geworden.

$-45-$

Wirft man ein Blick auf die Libretti verschiedener Autoren nach 1703, so wird man feststellen, dass sich italienische Arien in deutschen Opern großer Beliebtheit erfreuten. Nahezu jeder Librettist Hamburgs übernahm diese Praxis. Betrachtet man dagegen Wertungen, wie Haufe sie vornimmt, so mag man meinen, dass auch noch in jüngerer Zeit die ablehnende Haltung gegenüber der Italienisierungspraxis nicht gewichen ist:

"Eine weitere Verstärkung des italienischen Einflusses muss seit 1703 festgestellt werden, als [...] italienische Arien in die deutschen Textbücher eindrangen. Hinrich Hinsch begann damit in 'Die verdammte Staats-Sucht Oder der verführte Claudius'; das deutsche Libretto enthielt elf italienische Arien. Die italienische Oper nistete sich gewissermaßen auch in den deutschen Originalstücken selber ein. Es war der verhängnisvollste Schritt der Überfremdung, der innerhalb der Gesamtentwicklung zum auch künstlerischen Ruin der Hamburger Oper führen musste." 62

$$
-46-
$$

Was die Einfügung italienischer Arien angeht, malt Haufe ein äußerst düsteres Bild, meint beispielsweise, dass sogar in "[...] mythologischen Opern [...] diese üble Sitte [einzog]." ${ }^{63}$ Der Autor nimmt dabei für die Orpheus-Version von 1709 Keiser als Urheber der italienischen Texte an:

"Selbst Neubearbeitungen wie die von Bressands 'Orpheus'-Oper von 1709 und von Postels 'Ariadne' von 1722, die beide im einstigen Original keinen italienischen Vers besaßen, wurden Opfer dieser Manier. In beiden Fällen war Reinhard Keiser nicht nur der musikalische, sondern auch der textliche Neubearbeiter." 64

61 Wolff: Barockoper. S. 375.

62 Haufe: Mythologie. S. 100: Zu berücksichtigen ist dabei allerdings, dass es sich bei der Arbeit von Haufe um eine Dissertation aus dem Jahre 1964 handelt, die erst 30 Jahre später herausgegeben wurde. 
Dies scheint, was die musikalische Neubearbeitung angeht, im Falle des Orpheus von 1709 jedoch höchst zweifelhaft zu sein, berücksichtigt man die Quellenstudie Schulzes, in der der Autor über die Orpheus-Version von 1709 schreibt:

\begin{abstract}
"Es ist kaum wahrscheinlich, dass zu dieser Umarbeitung, die Keiser selbst vorgenommen haben mag, eine völlig neue Musik geschrieben wurde, vielmehr werden [...] die Arien, die textlich beibehalten wurden, wenigstens zum Teil auch musikalisch unverändert geblieben sein. Neukomponiert haben wird Keiser nur die zehn eingelegten italienischen Arien und vielleicht auch die Rezitative." ${ }^{65}$
\end{abstract}

$$
-48-
$$

Was den Ursprung der Italienisierungspraxis angeht, scheint sich die Mehrzahl der Autoren in der Zuschreibung klar auf Hinsch fixiert zu haben. Die Umarbeitung des Hamburgischen Librettos wird dagegen mehrfach Keiser selbst zugeschrieben, was auch wahrscheinlicher ist, denn offensichtlich trat wegen verschiedener Umstände, die Zelm beschreibt $^{66}$, zwischen 1706 und 1709 eine Pause in der Zusammenarbeit mit den Librettisten ein, so dass Keiser selbst zum Verfasser seines Textbuches werden bzw. auf die Bressandsche Vorlage zurückgreifen und sie bearbeiten musste.

$$
-49
$$

Haufe, der die Einfügung italienischer Arien als ersten "verhängnisvollen Schritt der Überfremdung" ${ }^{\prime 67}$ ansieht, kann, betrachtet man seine Arbeit als Gesamtheit, nicht überzeugen. Einerseits berücksichtigt er insbesondere den Hamburgischen Publikumsgeschmack, andererseits wirft Haufe Keiser und Hinsch vor, die Verfallsgeschichte der Gänsemarktoper eingeleitet zu haben. Der Autor fixiert sich in seiner Vorwurfshaltung rein auf den Einfluss der italienischen Sprache und vergisst dabei den ebenfalls wichtigen Einfluss der französischen Oper. Koch hebt in einem Aufsatz über die französischen Einflüsse auf Keiser hervor, dass gerade die erfolgreichste Oper des Komponisten (Carneval von Venedig ${ }^{68}$ ) durch André Campras Opéra-ballet Le carnaval de Venise (Uraufführung 1699) angeregt wurde. ${ }^{69}$ Vereinzelt

\footnotetext{
65 Schulze: Quellen. S. 36.

Zelm: Opern. S. 32.

Haufe: Mythologie. S. 62.

Schröder vermutet, dass Keisers Carneval von Venedig ca. 67 mal in Hamburg gespielt wurde, siehe: Koch, Klaus-Peter: Reinhard Keisers Schaffen im Hinblick auf französische Einflüsse. Französische Einflüsse auf deutsche Musiker im 18. Jahrhundert. Hg. Friedhelm Brusniak und Annemarie Clostermann. Köln: Studio, 1996. S. 89. Ebd. S. 81.
} 
findet man sogar Opern, in deren Libretti drei Sprachen Eingang gefunden haben, so beispielsweise in Johann David Heinichens Die Römische Großmuth/ Oder Calpurnia (Textdichter: Übersetzung von Johann Ulrich König nach italienischer Vorlage von Grazio Bracciolis Mario, Venedig 1713). Dieses Werk enthält neben deutschen und italienischen auch zwei französische Arien. ${ }^{70}$

$-50$

Die vielfältigen ausländischen und besonders auch die volkstümlichen Einflüsse trugen sicherlich zu dem Erfolg der Hamburgischen Oper bis 1738 bei und sind nicht als eine plump opportunistische Übernahme von Elementen, die sich anderswo schon als erfolgreich bewiesen hatten, anzusehen, was auch Wolff zusammenfassend bemerkt: "Man wollte in Hamburg dem breiten Publikum entgegenkommen und tat dies nicht in einer billigen, tiefstehenden Weise (was man bisher irrtümlich oft annahm), sondern man bemühte sich in jeder Hinsicht um einen künstlerisch hochstehenden Opernstil $[\ldots] .{ }^{11}$

$$
-51-
$$

Der Autor sieht nicht in dem "Überhandnehmen des italienischen Einflusses", sondern in der neuen, rationalistischen Geisteshaltung der Zeit den Grund für das baldige Ende der Hamburgischen Oper. ${ }^{72}$ Vor allem Johann Christoph Gottsched war es, der das unnatürliche Moment der Oper ablehnte. Er wandte sich "[...] gegen die Wortwiederholungen, die Vergleiche, gegen das Maschinenwesen der Oper und gegen die Verwendung der italienischen Sprache [... $]^{173}$, postulierte im Gegenzug dafür die Handlungsorientierung an der Natur. Gottscheds Forderungen und bestimmte Passagen aus den Schriften Barthold Feinds, die die Verteidigung der "bewussten Unwirklichkeit" der Oper intendieren, können diesbezüglich gewissermaßen als paradigmatische Gegenpositionen gelten.

$$
-52-
$$

Kommen wir auf unseren Vergleich der beiden Libretti zurück, so kann man nicht nur feststellen, dass in der späteren Version italienische Arien eingefügt wurden, auch die Bezeichnung Aria taucht (bei größtenteils gleich lautendem Arientext) erst im Libretto des Jahres 1709 auf, während wir diese Gattungsbenennung in der 1698er-Fassung vergeblich suchen. Das mag daran liegen, dass die Da-Capo-Arie (alle eingefügten italienischen Arien sind Da-Capo-Arien), die sich in Deutschland um 1700 als Gattung

Marx: Katalog. Nr. 233.

Wolff: Barockoper. S. 375.

Ebd. S. 341.

Ebd. S. 341-42. 
gegenüber der Strophenarie etabliert hatte ${ }^{74}$, 1709 emphatischer als noch 1698 als wichtiges italienisches Gattungsvorbild innerhalb der Oper angesehen wurde ${ }^{75}$, wofür die Keisersche Einfügung italienischer Arien zusätzlich spricht.

$$
-53-
$$

Hieraus lassen sich erneut Rückschlüsse auf die Ansprüche des Hamburgischen Publikums ziehen. Offensichtlich wollte der Opernbesucher darüber informiert sein, wann man sich innerlich auf eine affektgeladene Arie in italienischer Sprache einzustellen hatte, um sich an ihr richtig "ergetzen"76 $\mathrm{zu}$ können. Dafür eignete sich freilich die besondere Kennzeichnung der entsprechenden Arien im Textbuch. Dass die italienischen Arien im Mittelpunkt des Interesses standen, bezeugt jedenfalls Marx: "Direkten Einblick in den Geschmack eines zeitgenössischen Hörers gestatten zwei Bücher aus der Weimarer Sammlung (Salomon, 1703; Lucretia, 1705), deren Eigentümer die Qualität der Arien mit Anzeichnungen von ' + ' bis ' +++++ ' beurteilte. ${ }^{177}$

$$
-54-
$$

Das zentrale Gewicht lag also auf den Arien. Es empfahl sich, sie im Textbuch zu kennzeichnen, denn offensichtlich war das Interesse nicht immer nur der Opernhandlung zugeneigt:

\begin{abstract}
"Additionen von Geldsummen, Zinsberechnungen, Kalenderdaten und ähnliche Notizen in den Textbüchern belegen, dass das Geschehen auf der Bühne nicht immer im Mittelpunkte des Interesses stand. Das Theater war vor allem ein Treffpunkt der Gesellschaft, und so hatte das handliche Heft mitunter auch als 'postillon d'amour' zu dienen wie das Hamburger Exemplar des Esther-Textes [...], dessen letzte, blanke Seite die Botschaft 'dein, dein, mein hertz' trägt. ${ }^{178}$
\end{abstract}

\footnotetext{
74 Leopold, Silke: Art. Arie [II. 17. Jahrhundert]. MGG. Zweite, neubearbeitete Ausgabe. Hg. Ludwig Finscher. Sachteil. Bd. 1. Kassel: Bärenreiter; Stuttgart: Metzler, 1994. Sp. 812-816. Sp. 813-14.

75 Die Da-Capo-Arie (als aus Italien importierte Gattung) wurde besonders von Christian Friedrich Hunold (genannt Menantes) durch seine Schrift Theatralische / Galante und Geistliche Gedichte (1706) unterstützt. Vgl. Smart, Sara: Die Oper und die Arie um 1700. Zu den Aufgaben des Librettisten und zur Form und Rolle der Arie am Beispiel der Braunschweiger und Hamburger Oper. Studien zum deutschen weltlichen Kunstlied des 17. und 18. Jahrhunderts. Hg. Gudrun Busch und Anthony J. Harper. Amsterdam: Rodopi, 1992. S. 183-212. S. 194. 


\section{Unterschiedliche Szenenanweisungen und ihre bühnentechnische Umsetzung}

$-55-$

Prinzipiell unterscheiden sich die Schlussszenen der beiden hier verglichenen Libretti nicht: Der Sonnengott Phœbus kommt zu guter Letzt als schlichtende Instanz vom Himmel, fügt das Paar Aristeus und Autonoe wieder zusammen und spricht: "Damit des Orfeus angedenken verewigt sey / drum wolt ich unter der gestirne Reih die Stelle seiner Leyer schenken. Erkennt hieraus / wie Tugend / Kunst und Wissen der himmel selber zu belohnen ist beflissen." Die dazugehörigen Auftrittsanweisungen in den Libretti sind allerdings unterschiedlich. Im Textbuch von 1698 findet man als Kommentar zu der entsprechenden Szene: "Des Föbus prächtiger Pallast kömmt vom Himmel herunter" und: "Der gantze Schau=Platz verändert sich in des Föbus Pallast." Die Szenenanweisung des Librettos von 1709 verlangt dagegen eine andere Art der prächtigen Götterdarstellung: "Phoebus in einer vortrefflichen Machine." Diese Machinen tauchen (zumindest in dieser Bezeichnung) in Libretti der Braunschweiger Oper noch nicht auf, sie sind als Theatereffekt typisch für die Gänsemarktoper in Hamburg. ${ }^{79}$

$$
-56-
$$

Betrachtet man des weiteren die Szene, in der der Zauberer Zarxis (auf Befehl der Intrigantin Thya) eine Schlange hervorzaubert, die Eurydice vergiften soll, so fällt auf, dass diese Szene in der späteren Version an den Anfang der dritten Handlung gesetzt wurde. Hier befand sie sich in der Version des Jahres 1698 noch nicht, der Zauberer erscheint dort erst im zweiten Auftritt. Zusätzlich tritt in der späteren Fassung nur e i n Zauberer auf, im jüngeren Textbuch finden wir dagegen die Szenenanweisung: "Zarxis, Thya und e t lic he Zauberer hernach" (Hervorhebung von mir). Wiederum lässt dies Rückschlüsse auf die Einstellung des Publikums zu, das in Hamburg offenbar besonders emphatisch "afficirt" ${ }^{80}$ werden musste.

$$
-57-
$$

Nach dieser Szene heißt es in beiden Textbüchern: "Zarxis macht unterschiedliche Kreise und Zaubereyen." Die folgende Anweisung findet sich aber wiederum nur im Libretto von 1709: "Unter währender Aria [Drey beköpffte Hecate] kommen verschiedene Furien aus der Erden mit Fackeln / welche eine Schlange mit sich bringen / und mit denen übrig einschreitenden Zauberern tanzen."

Wolff: Barockoper. S. 29.

Feind: Gedicht. S. 108. 
Keiser verstärkte die Wirkung der Zauberszene also nicht nur dadurch, dass er dem Hamburgischen Publikum eine ganze Gruppe von Magiern, die er zudem noch einen Tanz aufführen ließ, präsentierte. Durch die mit Fackeln bestückten Furien, die aus dem unteren Teil der Bühne empor gezogen wurden, gelang eine weitere Intensivierung, die aufgrund der hervorragenden technischen Bedingungen des Opernhauses in Hamburg gut zu realisieren war. ${ }^{81}$

\section{Abschließende Bemerkungen}

$-59-$

Obwohl mythologische Stoffe mit Beginn des 18. Jahrhunderts in Hamburg hinter der Beliebtheit historischer (z. B. Croesus ${ }^{82}$, 1711) und aktueller Sujets (z. B. Die Leipziger Messe $\left.^{83}, 1710\right)$ zurücktraten ${ }^{84}$, war es Keiser durchaus möglich, eine solche Oper mit Erfolg aufzuführen. Zwar ist nur eine Vorstellung der Oper Die Biß in / und nach dem Todt / unerhörte Treue / Des ORPHEUS von 1709 sicher belegt $^{85}$, doch liegt dies wohl nicht an dem geringen Zuspruch, den dieses Werk bekam, sondern lediglich an der Tatsache, dass Aufführungen der Hamburger Oper erst ab 1719 ausführlicher dokumentiert sind. ${ }^{86}$

$$
-60-
$$

Ziel dieser Ausführungen war, die wichtigsten Veränderungen der Orpheus-Vorlage Bressands durch Keiser $\mathrm{zu}$ benennen und sie mit den sich wandelnden opernästhetischen Voraussetzungen, die besonders in den Schriften Barthold Feinds zum Ausdruck kommen, zu begründen.

$$
-61-
$$

Wir sahen, dass italienische Arien für die Aufführung des Werkes im Jahre 1709 eingefügt wurden, um den Affektgehalt zu erhöhen. Zudem verstärkte Keiser durch seine Bearbeitung die Wirkung bestimmter "Actiones" und ließ umfangreichere Sprechpassagen streichen, weil sie an der Hamburger Gänsemarktoper zu diesem Zeitpunkt als veraltet galten und man das Publikum nicht "auf dem Theatro [...] frieren" ${ }^{87}$ lassen wollte.

Wolf: Barockoper. S. 355/357.

Marx: Katalog. Nr. 161.

Ebd. Nr. 53.

Haufe : Mythologie. S. 104.

Marx: Katalog. S. 474.

Ebd. S. 467 f.

Feind: Gedichte. S. 106. 


$$
-62-
$$

Festzuhalten ist, dass die mehrfache Beschäftigung Keisers mit dem Orpheussujet ein Beleg für die Beliebtheit dieses Stoffes in der frühen deutschen Oper ist. Es würde sich lohnen, dies einmal wissenschaftlich ausführlich aufzuarbeiten, denn offensichtlich bot gerade die Be- und Verarbeitung des Orpheus-Mythos viele Möglichkeiten, um Moralarien $^{88}$ an- und "geschickte[...] Intruigen [...]"89 in die Bühnenhandlung einzubringen, um so auch im kühlen Norddeutschland "[...] das Auditorium zu afficiren" $"$. 